\title{
Measurement of longitudinal acceptance and emittance of the Oak Ridge Spallation Neutron Source Superconducting Linac
}

\author{
Y. Zhang, ${ }^{*}$ J. Galambos, and A. Shishlo \\ Oak Ridge National Laboratory, P.O. Box 2008, Oak Ridge, Tennessee 37831-6461, USA
}

(Received 19 May 2008; published 8 October 2008)

\begin{abstract}
The longitudinal acceptance of the Spallation Neutron Source superconducting linac is computed with a longitudinal model. A beam current monitor and beam loss monitors are utilized in a new beam acceptance measurement technique, and the measured results show close agreement with the model. Based on the simulations and on the measurements of the superconducting linac acceptance, we developed a novel method to measure beam bunch shape, beam energy profile, and the longitudinal emittance at the entrance of the linac. The experimental measurements reveal that a large longitudinal beam halo exists in the injected beam to the superconducting linac, and the longitudinal rms emittance is approximately twice that of the nominal design. The simple measurement method is applicable to other superconducting linacs.
\end{abstract}

DOI: $10.1103 /$ PhysRevSTAB.11.104001

PACS numbers: 29.20.Ej

\section{INTRODUCTION}

The Spallation Neutron Source (SNS) is a short-pulse neutron scattering facility at Oak Ridge National Laboratory. The accelerator complex consists of a $2.5 \mathrm{MeV} \mathrm{H}^{-}$injector, a $1 \mathrm{GeV}$ linear accelerator, an accumulator ring, and associated beam transport lines. Most of the beam energy comes from a Superconducting Linac (SCL) of approximately 160 meters long-from $186 \mathrm{MeV}$ to $1 \mathrm{GeV}$. The linac produces a $1 \mathrm{msec}$ long, $38 \mathrm{~mA}$ peak, chopped beam pulse at $60 \mathrm{~Hz}$ for accumulation in the ring. In the baseline design, beam is accumulated in the ring over 1060 turns reaching an intensity of $1.5 \times 10^{14}$ protons per pulse, then extracted and transported to a liquid-mercury target for neutron production [1].

The SCL comprises 23 cryomodules with 81 independently powered niobium superconducting (SC) cavities. The design consists of 33 medium beta cavities with a geometrical beta of 0.61 that provide acceleration from 186 to $391 \mathrm{MeV}$, and 48 high beta cavities that provide acceleration from 391 to $1000 \mathrm{MeV}$. The medium beta cavities have a design gradient of $10.2 \mathrm{MV} / \mathrm{m}$ and the high beta cavities have a design gradient of $15.9 \mathrm{MV} / \mathrm{m}$. In practice, the cavities have a large spread in gradients, ranging from 8 to $18 \mathrm{MV} / \mathrm{m}$, and many high beta cavities are below the design gradient. In addition, typically several SC cavities are disabled for various reasons. The SCL cavity average synchronous phase is set at $-20.5^{\circ}$ for the cases discussed here.

Because of the rf superconductivity technology and the high intensity beams, conventional beam interception diagnostic devices could contaminate the cavity delicate surfaces, and are not acceptable in the SCL. Laser wire transverse beam profile monitors are available at several

\footnotetext{
*zhangyn@ornl.gov
}

selected locations in the SCL, but no longitudinal beam profile instruments are installed. In recent neutron production operations, some beam loss has been observed in the SCL that may be a longitudinal issue. Measurements of the SCL longitudinal acceptance, the injection beam emittance, and the longitudinal beam halo become important to understand the cause of the beam halo, and to mitigate the beam loss in the superconducting linac.

A simple linac longitudinal transport model is developed to compute the longitudinal acceptance. The SCL acceptance is measured using beam transmission determined by nonintercepting devices - a beam current monitor (BCM) and the beam loss monitors (BLMs), in conjunction with phase and energy scanning techniques. The beam acceptance measurements have sharp boundaries which are utilized as virtual beam shutters, so that beam phase and energy profiles can also be reconstructed. From the BCM data, beam bunch shape, beam energy profile, and the longitudinal emittance are measured, and in the BLM measurements, beam longitudinal tails (or halos) are shown. The beam acceptance measured with the BCM and BLMs is close to the model prediction.

\section{SIMULATION}

The longitudinal beam acceleration model of the SCL cavity is based on a standard drift-kick-drift thin lens approximation [2] in which a kick is applied at each cavity cell. The cavity gradients used here are based on beam based calibrations [3] and the cavity synchronous phases are determined using beam time-of-flight signature matching (or more specifically, phase-scan signature matching [4]) measurements. In the phase-scan signature matching the measured "signature" of the change in the downstream beam arrival time with variations in an upstream rf cavity is matched with model results by adjusting the cavity amplitude, synchronous phase, and input beam energy. Details of the models are given in Refs. [5,6]. 
The acceptance calculations use the longitudinal model described above. Particles are launched from the beginning of the SCL with various phase and energy errors with respect to (w.r.t.) the design values of $186 \mathrm{MeV}$ input energy and $-20.5^{\circ}$ synchronous phase. All particles are tracked to the end of the SCL. If the final beam energy deviates from the design value by more than a specified cutoff, it is defined to be outside the linac acceptance, otherwise it is considered inside the acceptance. The energy cutoff from the design output energy is dependent on the particular beam requirements (e.g. inside the longitudinal separatrix of the SC cavity, and cleanly transported through the SCL, etc.).

Figure 1 shows the longitudinal acceptance of the SCL for two cases, one using an average synchronous phase of $-17.4^{\circ}$ for each medium beta cavity and another using $-20.5^{\circ}$. The acceptance boundary is defined here as containing the input beam phase space beyond which the final output beam energy is more than $12 \mathrm{MeV}$ off the design SCL output energy. This energy value roughly corresponds to the requirement for injection into the SNS Ring, so that all beam outside of this space will be completely lost in the SCL and in the downstream accelerator systems. For comparison, an SCL injection beam emittance created by tracking a waterbag distribution with 6 times the nominal emittance from the rf quadrupole to the SCL entrance is also shown. The factor of 6 is used to exaggerate the size of the input beam emittance. Even with this large input beam emittance, it is still small compared to the longitudinal acceptance. However, when particles fall out of the SCL longitudinal acceptance, as in the case of halo particles, they are no longer accelerated in the linac, and not matched to the downstream quadrupole lattice which is designed for the fully accelerated particles, and consequently, they are

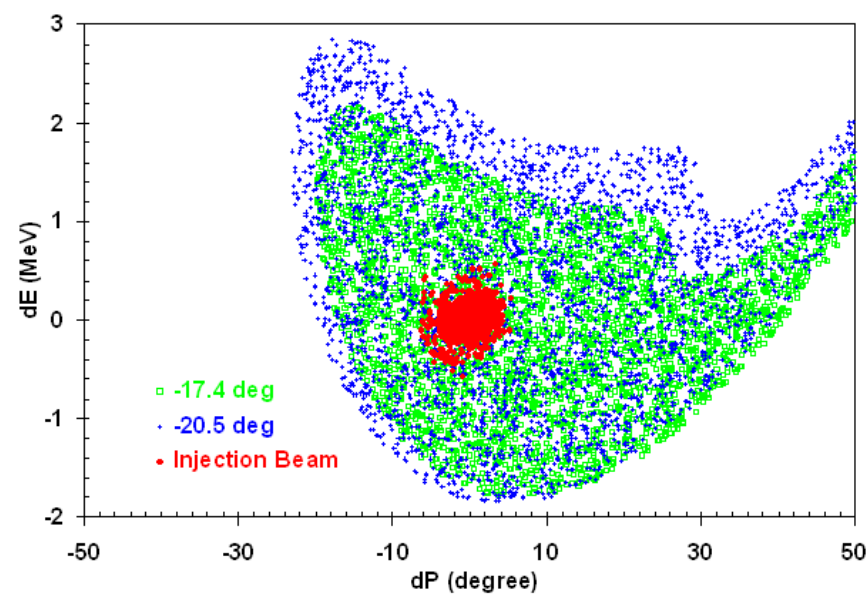

FIG. 1. (Color) Longitudinal acceptance of the SCL with an average synchronous phase of $-17.4^{\circ}$ (green squares) for each medium beta cavity and $-20.5^{\circ}$ (blue dots), an injection beam emittance with tails 6 times greater than the nominal beam is shown (red dots) for comparison. lost in the SCL due to transverse mismatch [7]. Here the limiting aperture radius of the SCL is approximately $4 \mathrm{~cm}$.

\section{LONGITUDINAL ACCEPTANCE MEASUREMENT TECHNIQUE}

When the beam falls out of the longitudinal acceptance, it is expected to be lost in the SCL. Using this fact, one can measure the transmission fraction of the beam with a BCM at the exit of the linac, as a function of the input beam energy and synchronous phase w.r.t. the design values. In addition, one can use beam loss measurements with all BLMs in the SCL to determine the relative fraction of beam that is lost for various input phase and energy conditions.

A primary component of the acceptance measurement is varying the beam energy and phase about the design values at the start of the SCL. We prefer to not vary the upstream normal conducting linac rf settings to affect the SCL input beam phase and energy manipulations, because the upstream copper structures are coupled cavity linac (CCL) structures with 96 accelerating gaps per cavity, and any phase or amplitude deviations from the design values can have a detrimental effect on the output beam emittance as well as the output energy and phase. Instead we rely solely on SCL cavity manipulations.

For the phase variations about the design synchronous phase, we simply shift all the cavities by the same absolute value using the cavity rf control system. This is equivalent to varying the beam phase at the start of the linac, while retaining the same synchronous phase setting relationship from one cavity to the next.

For energy variations the situation is slightly more complicated. We use the first SCL cavity to produce the input beam energy changes. Instead of setting the first cavity to the nominal phase, it is initially set to $-90^{\circ}$. When the beam energy exiting the first SCL cavity is changed, the arrival time at the downstream cavity is slightly changed. This requires an adjustment (rephasing) of the downstream cavities phase settings to recover their original synchronous phases. This is done using a model based phase scaling technique [8]. With this initial setup the first cavity's phase can be varied from $-180^{\circ}$ to $0^{\circ}$ to provide $\sim \pm 7.7 \mathrm{MeV}$ beam energy variation into the second cavity, which is sufficient for the acceptance measurements.

Figure 2 shows the model predicted cavity phase changes for two different cases: (1) only the first cavity phase is shifted by 10 degrees and (2) the injection beam energy is adjusted by $0.6 \mathrm{MeV}$ without an appropriate rephasing of the superconducting linac rf. The change in the synchronous phase from the design along the downstream SCL cavities starting at cavity 2 is shown for both of these cases. In both cases, the design synchronous relationships in the linac are broken (cavity synchronous phase varies from the design $-20.5^{\circ}$ ). Note that for the case of the injection energy change there is a slight change 


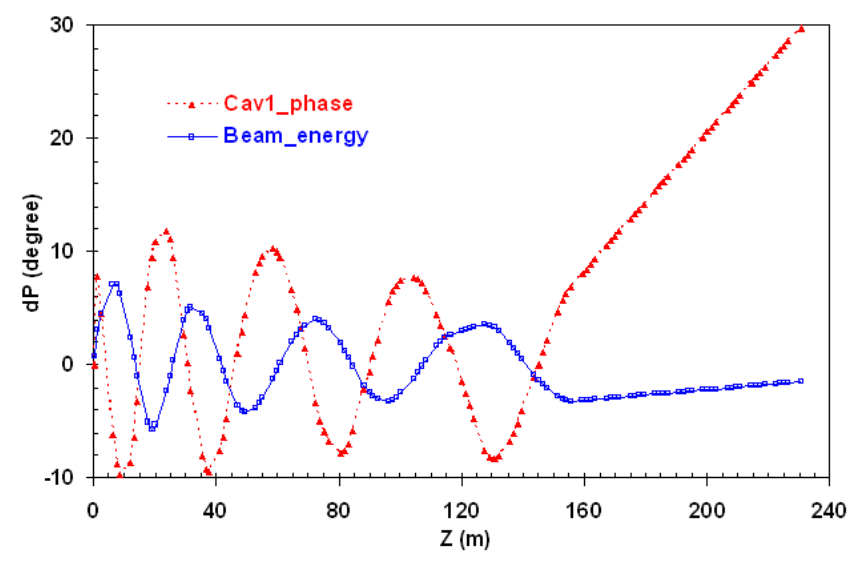

FIG. 2. (Color) Model predicted change in downstream cavity phase without rephasing the superconducting linac when (1) only the first cavity phase is shifted by 10 degrees (Cav1_phase), and (2) the injection energy is changed by $0.6 \mathrm{MeV}$ by adjusting the upstream cavity one phase (Beam_energy).

( $\sim 1$ degree) in the synchronous phase at the first downstream cavity (SCL cavity 2 in this case). It is this slight change that is adjusted for in the rephasing of the SCL linac cavities when we vary the input beam energy. We investigated these phase oscillations and compared to measurements previously (called a phase damping study) [9]. The study demonstrated that the modeled phase oscillations shown here are similar to measurements, which is important since a model is used for some of the rephasing discussed here.

Energy scans can be performed at a selected phase by varying the first cavity's phase to enact a desired energy change and adjusting the downstream cavity phases to recover the original synchronous phase settings using the technique described in Ref. [8]. The basic technique used in Ref. [8] is a beam arrival time difference method. First, the cavity phases for each SCL cavity are set with a beam based technique, as in a normal machine tune-up procedure. This essentially represents a measurement of the beam arrival time at each cavity w.r.t. the rf. If an upstream cavity is adjusted so that its energy gain is modified, a model can be used to predict the change in the energy gain and subsequent changes in beam arrival times downstream. Each change in a beam arrival time at a cavity is equivalent to a change in its synchronous phase and can be appropriately used in modeling the change in the beam acceleration through the SCL. For the case of an energy scan at "fixed phase" described here, one adjusts the downstream cavity rf phase setpoints to compensate for the change in arrival time and recover their original synchronous phase. Once a reliable reference set of beam based cavity phase measurements are acquired in the original linac tune-up, use of a model based difference technique to manipulate downstream cavity settings is fast and accurate.

The longitudinal acceptance scan is done starting at the second SC cavity. Thus all acceptances reported here are

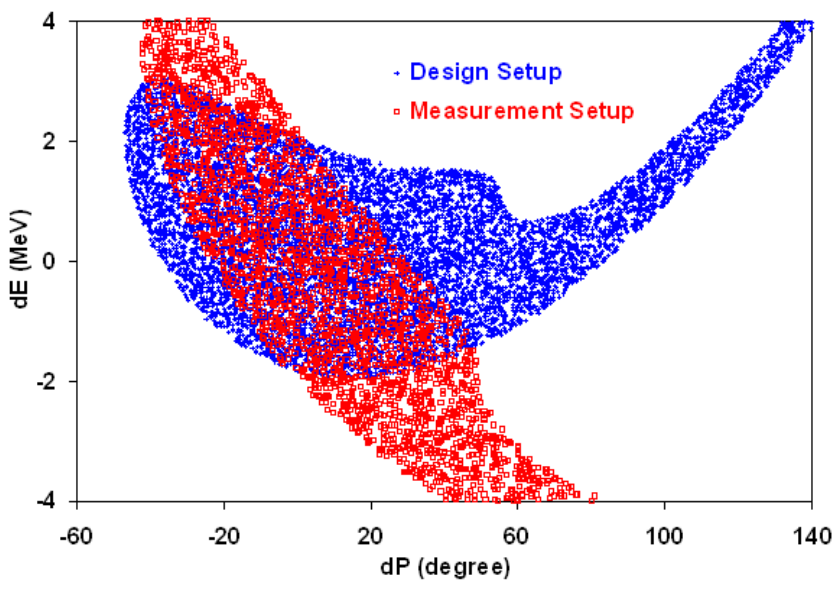

FIG. 3. (Color) Model longitudinal acceptance of the first cavity at the design phase $-15^{\circ}$ (Design setup) and at the measurement phase $-90^{\circ}$ (Measurement setup).

for the entrance to the second SCL cavity. The beam phase and energy can be rastered about the design values using these techniques, and the beam current transmission and/or the integrated beam loss through the entire superconducting linac can be used as measures of the longitudinal acceptance.

To understand the impact of measuring the acceptance at the entrance to the second cavity instead of the first one, we simulate the expected difference. Figure 3 shows the calculated acceptance to the first cavity for its nominal design setting and also for the case where it is set to $-90^{\circ}$, as in the experimental setup used here. Indeed, the acceptance is dramatically altered for this cavity. However, as described above, the measurements are done with respect to the entrance of the second cavity. The longitudinal acceptance for this cavity is shown in Fig. 4 for the case of cavity 1 set to the design phase of $-15^{\circ}$ and set to $-90^{\circ}$ for the

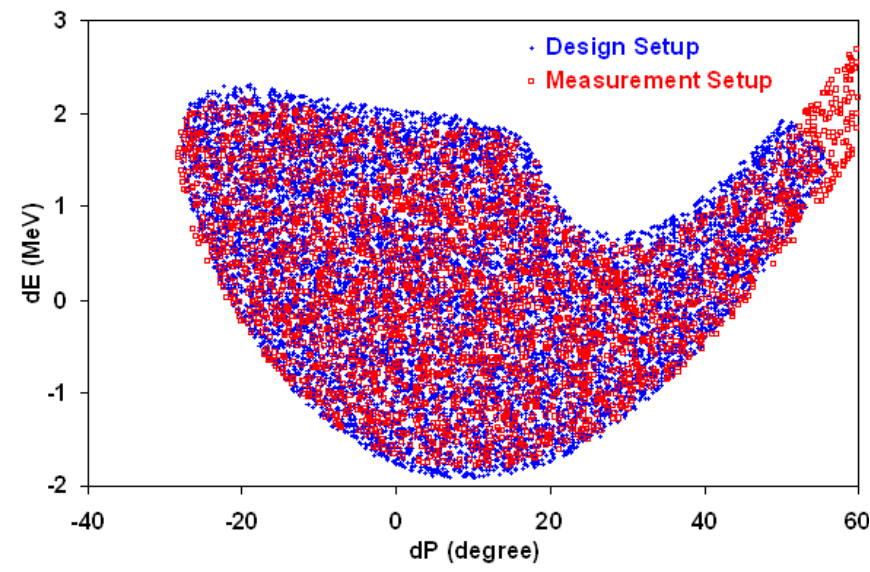

FIG. 4. (Color) Model longitudinal acceptance of the second cavity with the first cavity at the design phase $-15^{\circ}$ (Design setup) and at the measurement phase $-90^{\circ}$ (Measurement setup). 
measurement. In this case there is only a small difference, due to the small change in the initial beam energy at the entrance to cavity 2 from 193 to $186 \mathrm{MeV}$.

\section{ACCEPTANCE MEASUREMENT RESULTS}

A small beam intensity is used during the beam phase and energy scans described above for the acceptance measurements; as by definition, most of the beam will be lost as one approaches and crosses the acceptance boundary. The superconducting cavity is a delicate component and beam loss above several watts in a SC cavity could quench it, or cause other damage. The peak beam current in the measurements varies from 12 to $34 \mathrm{~mA}$ for a short pulse of only $600 \mathrm{nsec}$, and beam power dumped in the entire SCL is controlled to less than $2 \mathrm{~W}$ with an appropriate time interval between each beam pulse.

The SNS beam loss monitor (BLM) is a modified version of the well-known FNAL ion chamber, and there are 74 BLMs installed in the SCL. The beam current monitor $(\mathrm{BCM})$ is a fast current transformer which is installed at the exit of the linac. For detailed information of the beam diagnostics of the SNS accelerator systems, see Ref. [10]. In the measurement, we recorded the maximum beam loss and the sum of all the BLMs in the superconducting linac as well as the transmitted beam current. Though the total beam power dumped in the linac is less than $2 \mathrm{~W}$, the response of the BLM and the BCM is sufficient. Figure 5 shows a typical beam loss response throughout the SCL for beams inside and outside of the longitudinal acceptance. When the beam is outside the acceptance, the BLM response is 3-4 orders of magnitude higher than a fully transmitted beam within the acceptance.

So the longitudinal acceptance measurements can be performed with the BCM and with the BLMs. Figure 6

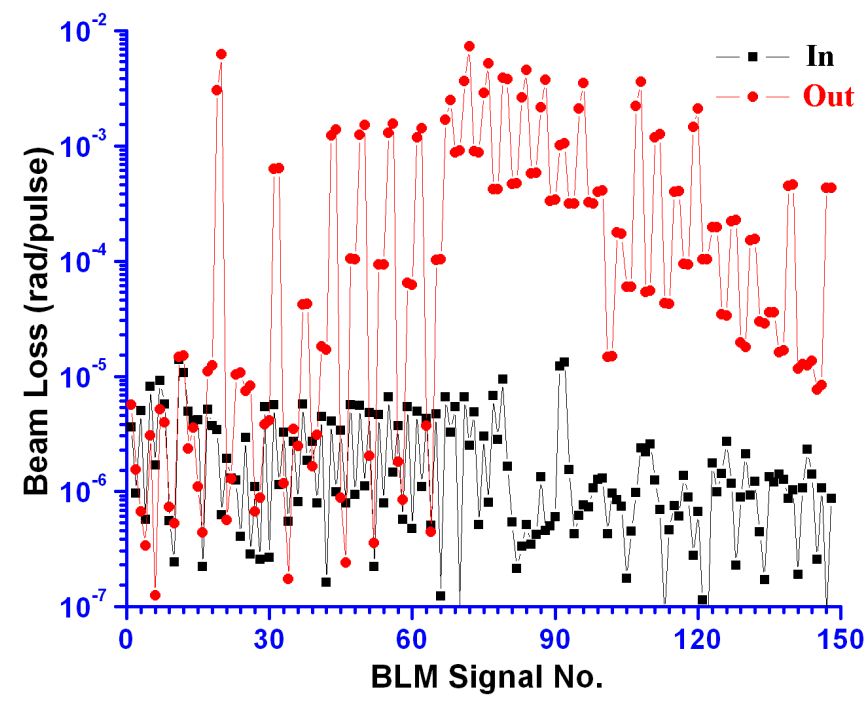

FIG. 5. (Color) Measured beam loss for beams within and out of the longitudinal acceptance. Each BLM recorded two signals, and beam power dumped in the SCL is approximately $0.5 \mathrm{~W}$.

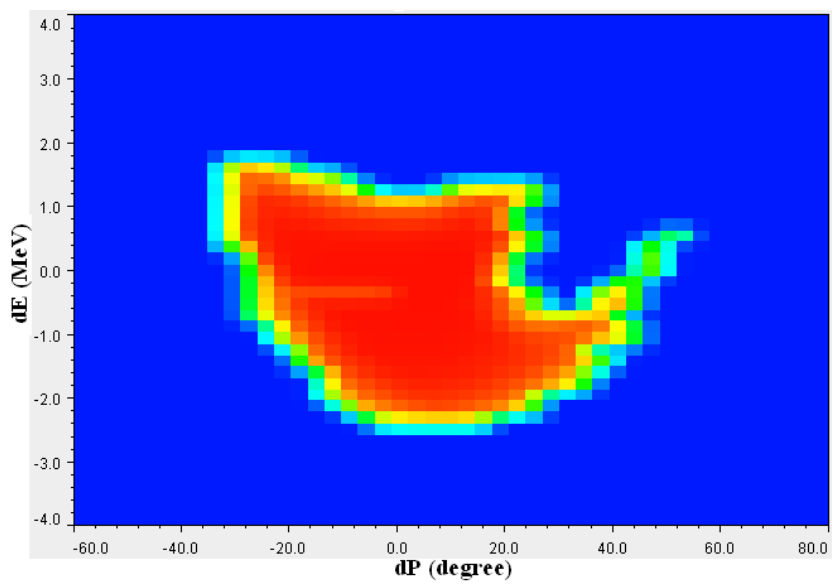

FIG. 6. (Color) Longitudinal acceptance measured at the second SCL cavity with the sum of all the linac BLMs.

shows the measured results of beam acceptance with the BLMs, and Fig. 7 is that with the BCM. In both these figures, the phase represents the difference from the design phase and the energy scale is the offset from the design energy at the entrance to the second SCL cavity. For Fig. 6, the color contours represent the sum of all the BLMs, and range from 0 to $0.4 \mathrm{rad} /$ pulse. For Fig. 7 the color contours represent different amounts of transmitted beam, ranging from 0 to $25 \mathrm{~mA}$.

Both BCM and BLMs acceptance measurements show qualitative agreement with the model as shown in Fig. 4, and the BCM results show good quantitative agreement. The longitudinal acceptance measured with BLMs for half the peak beam loss is smaller than that of the BCM measured $50 \%$ transmission. This is because the BLMs are saturated at high beam losses, and they are more sensitive to the tails of the beam than the BCMs. The BLMs easily respond to beam loss fractions of $10^{-4}$, whereas the BCMs are sensitive to only about a few parts in 100. In this sense the BLMs are a better measure of the

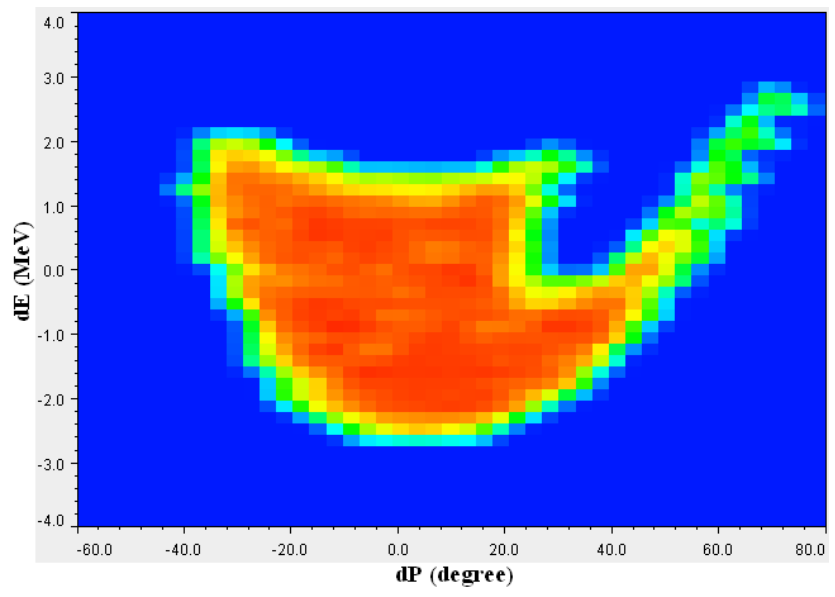

FIG. 7. (Color) Longitudinal acceptance measured at the second SCL cavity with the BCM at the exit of the SCL. 


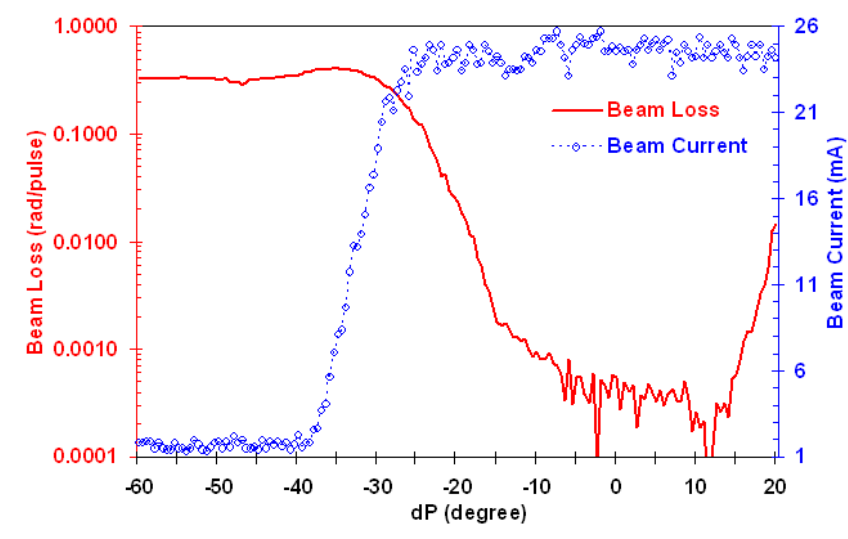

FIG. 8. (Color) The BCM signal measured in a beam phase scan, and the total beam loss measured with all BLMs. The design phase is 0 .

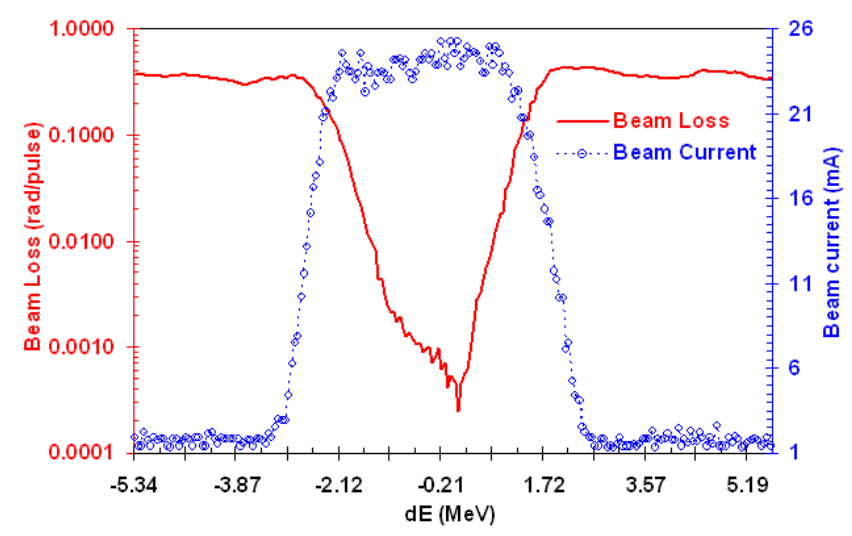

FIG. 9. (Color) The BCM signal and the total beam loss measured with all SCL BLMs in a beam energy scan.

beam halo, and the BCMs can be used as an indication of the core beam transmission.

While the phase scan does not give quantitative information about the absolute value of the amount of beam in the halo, it does show a phase tail $>40^{\circ}$ in Fig. 8. Scans performed in the beam energy direction show that the injection beam also has a large energy tail $>3 \mathrm{MeV}$, which is shown in Fig. 9, and size of the longitudinal beam halo is comparable with the linac acceptance. The BLMs show no response in these scans for cases beyond about $10 \%$ beam current reduction in the maximum BCM transmission because of saturation in the BLM devices.

\section{BEAM PROFILE AND EMITTANCE MEASUREMENT}

A clear variation of transmitted charge with injection beam phase and energy is shown in the scans of Figs. 8 and 9. This demarcation can be utilized as a shutter, to measure the bunch shape, beam energy profile, and the longitudinal emittance. A question arises: how thick is the acceptance boundary? This issue can be addressed with the longitudi-

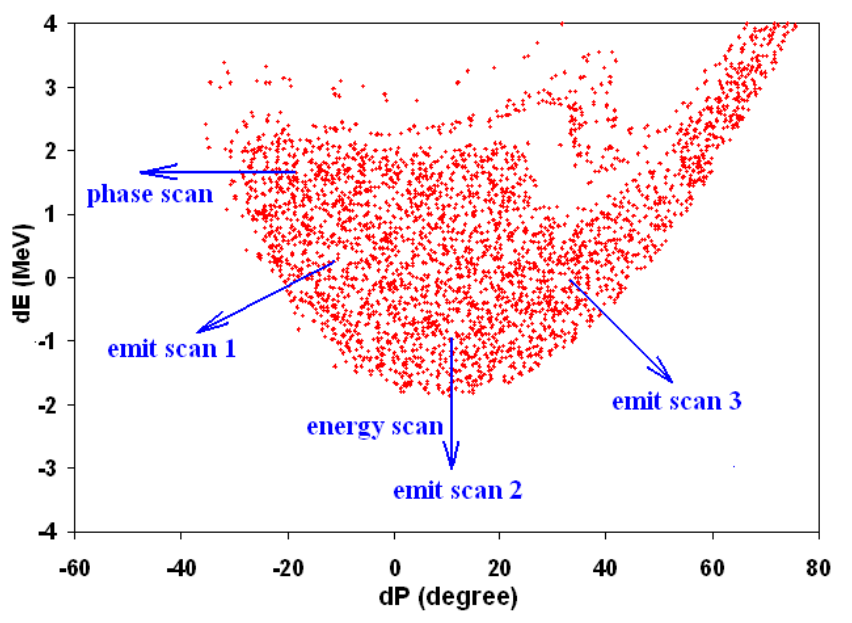

FIG. 10. (Color) Model of the longitudinal acceptance at the second SCL cavity with a $450 \mathrm{MeV}$ allowance for the output energy error. The diffuse upper boundary of the acceptance is not appropriate for beam profile measurement. The blue arrows show paths used for phase, energy, and emittance measurement scans.

nal acceptance simulation in Sec. II by very fine beam energy and phase changes across the demarcation. Figure 10 shows the calculated longitudinal acceptance for the case of the SCL (at the second cavity) cutoff energy being within $450 \mathrm{MeV}$ of the design output energy. Such a large energy error is considered here because it is still possible to transport off energy beam of this magnitude through the SCL as it has a large aperture and limited length. Note that even with this large allowable output energy error, the shape of the lower half of the longitudinal acceptance is similar to that shown in Fig. 4, which used only a $12 \mathrm{MeV}$ energy deviation cutoff for the acceptance definition. However, the upper boundary of this acceptance is more diffuse than the lower counterpart because of many tiny islands in longitudinal phase space in the upper region that allow beam acceleration within this large energy error criteria (which may nonetheless be transmitted to the measuring devices used here). An emittance measurement performed by scanning the beam across a boundary and measuring the transmission requires a sharp boundary relative to the size of the emittance being examined. Thus, for all the beam phase, energy, and emittance measurements discussed, we perform scans across the lower boundary. In particular, the lower boundary is very sharp and clean with phase width $<0.4^{\circ}$, and energy width $<0.05 \mathrm{MeV}$. It is sufficient for measurement of beam longitudinal profiles and emittance, as the linac beam has phase and energy jitter (approximately $0.5^{\circ}$ and 0.1 to $0.2 \mathrm{MeV}$ ) that are larger than this boundary layer. We note that the measured acceptance contours in Figs. 6 and 7 also indicate a more diffuse boundary in the region near $\Delta E=+2 \mathrm{MeV}$ and $\Delta$ phase $=+30$ degrees.

Figures 11 and 12 show the beam bunch shape and energy spread distribution measured with the BCM. The 


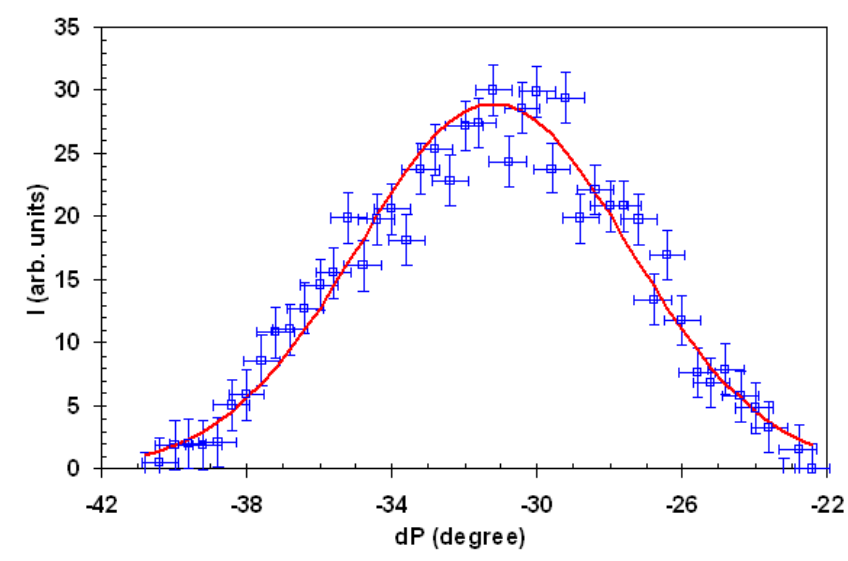

FIG. 11. (Color) Beam bunch shape measured at the second SCL cavity with the BCM in a phase scan, beam phase width (sigma) is approximately $3.8^{\circ}$.

measurement errors mainly come from beam current fluctuations, BCM noise, and beam phase and beam energy jitter. The phase and energy distributions are derived from scans taken along the paths shown in Fig. 10. The resultant profiles are fit with a Gaussian distribution (indicated in red in the figures) to derive the characteristic width.

The measured beam phase shown in Fig. 11 is taken by scanning the beam phase at constant energy as indicated by "phase scan" in Fig. 10. The resultant phase width is approximately $3.8^{\circ}$ - very close to an independent beam bunch measurement (of $3.7^{\circ}$ ) using a bunch shape monitor (BSM) in the SNS normal conducting linac [11] which is only a few meters upstream of the SC cavity. The expected design width of the phase is $2.7^{\circ}$. The energy profile shown in Fig. 12 is derived from scanning the beam energy at constant phase as indicated by "energy scan" in Fig. 10. The energy width indicated in Fig. 12 is $0.33 \mathrm{MeV}$, as compared to a design value of $0.29 \mathrm{MeV}$ for cavity 1 phase at $-90^{\circ}$.

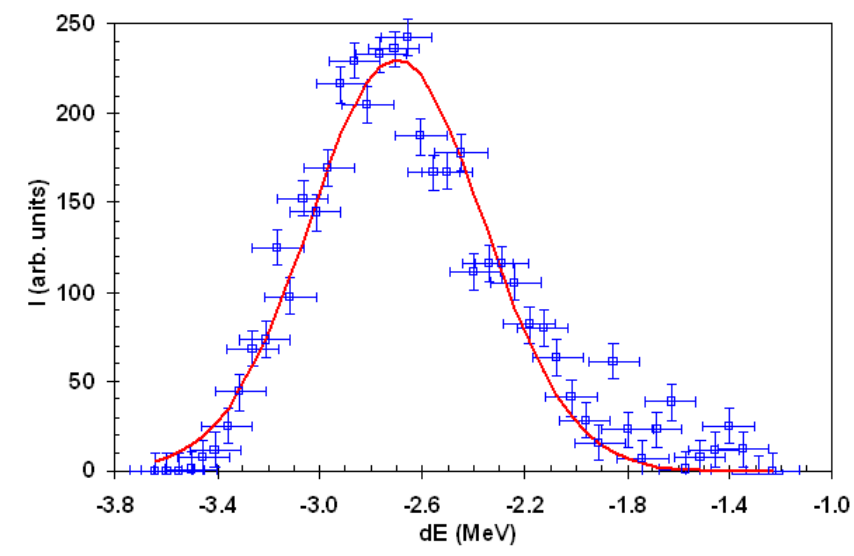

FIG. 12. (Color) Beam energy profile measured at the second SCL cavity with the BCM in an energy scan. The beam energy width (sigma) is approximately $0.33 \mathrm{MeV}$.
Using the natural shutter provided by the sharp acceptance boundary, extending the beam energy and phase profiles for a longitudinal emittance measurement for the high energy proton beams becomes a simple task. Several of these scans are performed across different paths in the longitudinal space, as indicated by "emit scan" paths in Fig. 10. The fractional BCM transmission contours in Fig. 13 indicate the relative amount of beam contained within these boundaries. The measured root-mean-square (rms) emittance is approximately $2.7 \mathrm{deg} \mathrm{MeV}$, about twice that of the nominal design, which is consistent with independent beam longitudinal emittance measurements with BSMs in the upstream normal conducting linac [12]. We noted that variations of the beam CourantSnyder parameters due to phase scan of the first SCL cavity in the longitudinal emittance measurement could be ignored, e.g., in simulations with IMPACT [13], for a phase scan of cavity one from $-95^{\circ}$ to $-85^{\circ}$ corresponding to a beam energy change of -0.7 to $+0.7 \mathrm{MeV}$ which covers the entire energy range used for the emittance measurement, the beam Courant-Snyder parameter change is less than $3 \%$ (emittance and beta change $<1 \%$, alpha changes approximately $2.6 \%$ in the simulation).

The errors of rms emittance measurements with the acceptance shutters for different linac systems depend on the beam energy, size and current fluctuations, linac rf properties, longitudinal model and the beam diagnostics involved, and there may not be a general rule. The resolution from beam jitter is about 0.5 degrees for the phase scan and about $0.2 \mathrm{MeV}$ for the energy scans (which leads to about $0.1 \mathrm{deg} \mathrm{MeV}$ resolution uncertainty for the emittance measurement). In addition there is a resolution in the BCM current of about $3 \%$ of the maximum current value, which contributes to uncertainty in the fraction of beam within an emittance contour. This effect has a larger absolute error for the case with small and large emittance

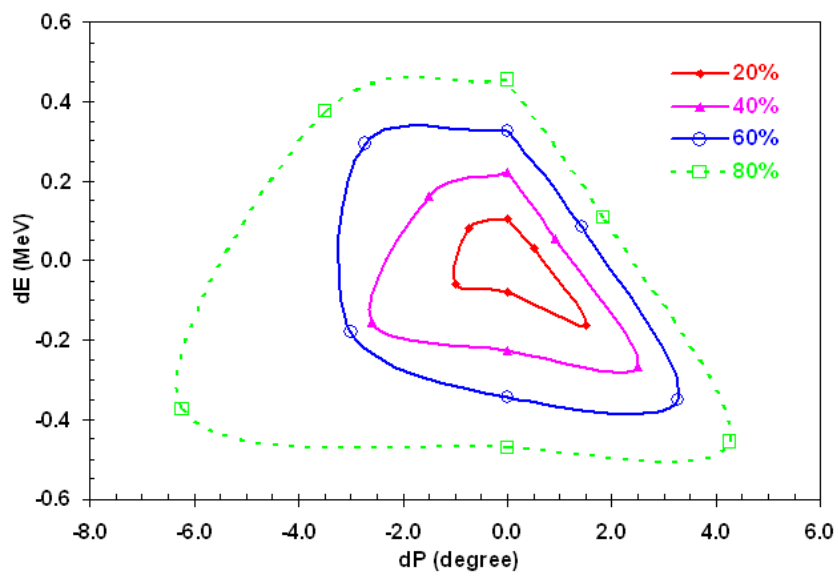

FIG. 13. (Color) One of the measured beam longitudinal emittance contours at the second SCL cavity, beam rms emittance is approximately $2.7 \mathrm{deg} \mathrm{MeV}$, or twice that of the nominal design. 


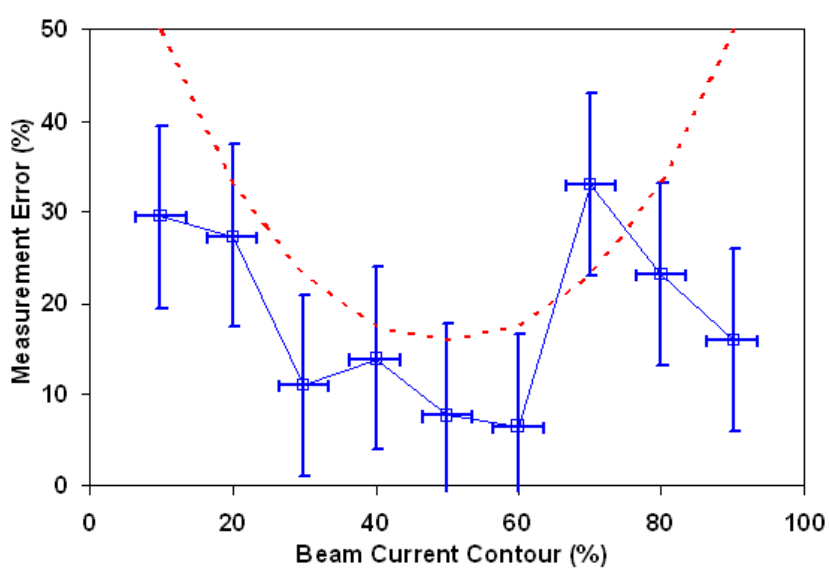

FIG. 14. (Color) Estimated (red dashed line) and measured (blue squares with error bars) errors of the measurements for different SNS beam contours.

extremes for which there is a relatively small amount of change in current transmission during the scans. Also there is an uncertainty introduced from the modeling used in enacting the energy scans, and we estimate this could lead up to a $10 \%$ error from the machine setup used for the scans used in the emittance measurement. Taken together, we estimate that these effects could result in about a $20 \%$ error in the emittance measurement for our case. Figure 14 shows for different SNS beam contours the estimated error from these effects (red dashed line). It also shows the rms difference in measured emittance fractions when the measurements are repeated 6 times (blue squares with error bars).

\section{CONCLUSION}

Techniques have been applied to measure the longitudinal beam parameters of the SNS superconducting linac. These techniques are possible in large part because of the flexibility in the operation of the SCL as it consists of many independently phased cavities. Measuring the beam transmission over beam phase and beam energy scans provides an acceptance boundary which closely agrees with the model predictions. Furthermore, the sharp boundary of the acceptance provides a natural shutter for beam bunch shape, beam energy profile, and the longitudinal emittance measurement. These measurements are in good agreement with independent BSM measurements. The simple method of longitudinal beam phase space analysis discussed in this study is applicable to other SC linac systems.

\section{ACKNOWLEDGMENTS}

The authors would like to thank A. Aleksandrov for many fruitful discussions and the BSM measurements which made this study possible.

[1] Stuart Henderson, in Proceedings Utilisation and Reliability HPPA, 2004, p. 257.

[2] B. Schnizer, Part. Accel. 2, 141 (1971).

[3] Y. Zhang et al., in Proceedings of the 2006 Linear Accelerator Conference, LINAC06, Knoxville, Tennessee, p. 315, http://accelconf.web.cern.ch/ AccelConf/106/PAPERS/TUP032.pdf.

[4] T.L. Owens, M. B. Popovic, E.S. McCrory, C.W. Schmidt, and L. J. Allen, Part. Accel. 48, 169 (1994).

[5] J. Galambos, A. Aleksandrov, C. Deibele, and S. Henderson, in Proceedings of the 2005 Particle Accelerator Conference, PAC05, Knoxville, Tennessee (IEEE, Knoxville, Tennessee, 2005), p. 1491.

[6] Y. Zhang and S. Henderson, Ref. [3], p. 423, http:// accelconf.web.cern.ch/AccelConf/106/PAPERS/ TUP073.pdf.

[7] Y. Zhang, S. Henderson, and D. Jeon, SNS Technical Note (2005).

[8] J. Galambos, S. Henderson, A. Shishlo, and Y. Zhang, Proceedings Utilization and Reliability HPPA, 2007.

[9] Y. Zhang, I.E. Campisi, and S.D. Henderson, Nucl. Instrum. Methods Phys. Res., Sect. B 261, 1036 (2007).

[10] http://neutrons.ornl.gov/diagnostics/diag_docs.html.

[11] A. V. Feschenko et al., in Proceedings of the 2007 Particle Accelerator Conference, Albuquerque, New Mexico, 2007 (IEEE, Albuquerque, New Mexico, 2007), p. 2608.

[12] A. Alexsandrov et al. (to be published).

[13] J. Qiang et al., J. Comput. Phys. 163, 434 (2000). 